\title{
Distribution of hepatitis C virus (HCV) genotypes in a Saudi Arabian hospital during the 2015-2020 period
}

\author{
Fadel Ali M. Almosa', Ali Hassan A. Alnasser², Jaffar A. Al-Tawfiq ${ }^{3,4,5}$ \\ ${ }^{1}$ Gastroenterology Unit, Department of Internal Medicine, Qatif Central Hospital, Ministry of Health, \\ Qatif, Saudi Arabia; \\ ${ }^{2}$ Department of Laboratory, Dhahran Eye Specialist Hospital, Ministry of Health, Dhahran, Saudi Arabia; \\ IInfectious Disease Unit, Specialty Internal Medicine, Johns Hopkins Aramco Healthcare, Dhahran, Saudi Arabia; \\ ${ }^{4}$ Department of Medicine, School of Medicine, Indiana University, Indianapolis, IN, USA; \\ ${ }^{5}$ Department of Medicine, School of Medicine, Johns Hopkins University, Baltimore, MD, USA
}

Article received 3 June, 2021; accepted 15 July, 2021

\section{SUMMARY}

Hepatitis $\mathrm{C}$ virus (HCV) is the leading cause of chronic liver disease. HCV genotypes and subtypes are important predictors of disease progression and antiviral treatment response. To our knowledge, there had been limited studies of HCV genotypes in Qatif, Saudi Arabia. This study aims to assess the distribution of HCV genotypes in Qatif Central Hospital, Qatif, Saudi Arabia. This is a retrospective study of adult patients with HCV infection between January 2015 and December 2020. Only patients with documented $\mathrm{HCV}$ genotyping were included. A total of $356 \mathrm{HCV}-$ infected patients fulfilled the inclusion criteria and were included in further analysis. Of those patients,
$179(50.3 \%)$ were males, and most were Saudi ( $\mathrm{N}=347$, $97.5 \%)$. The median age was 60 years, and $191(53.7 \%)$ were 50-69 years of age. Genotype 2 was present in 118 $(33.1 \%)$ of the patients, followed by genotype 4 in 92 $(25.8 \%)$, genotype $1 \mathrm{~B}$ in $62(17.4 \%)$, and genotype $1 \mathrm{~A}$ in $37(10.4 \%)$. The study showed that HCV genotype 2 is the predominant variant among chronic HCV patients in the study population. Monitoring the epidemiology of HCV genotypes may provide guidance in treatment decisions.

Keywords: HCV epidemiology, HCV prevalence, antiretroviral therapy, HCV viral RNA load.

\section{INTRODUCTION}

Tepatitis C virus (HCV) is a positive sin1 gle-stranded RNA virus of the genus Hepacivirus, belongs to the family Flaviviridae, with a high spontaneous mutation rate [1, 2]. The Middle East and North Africa (MENA) region has the highest incidence of HCV infection and has more than 15 million chronically infected patients [3]. However, the prevalence of $\mathrm{HCV}$ varies from region to region, ranging from $1.5 \%$ to $5.7 \%$ in Saudi Arabia [4, 5]. Chronic HCV infection accounts for

Corresponding authors

Ali Hassan A. Alnasser

E-mail: alialnassser@gmail.com
$20 \%$ of hepatocellular carcinoma (HCC), and about 700,000 people die annually from complications related to HCV [6].

$\mathrm{HCV}$ is classified into seven genotypes (GT) and 67 subtypes such as 1a, 1b, 2a, 3a, 4a [3, 7]. Globally, GT-1 is common in North America, South America, Western and Northern Europe, representing $46 \%$ of all HCV cases [7]. GT-3 is common in South Asia, Australia, and some parts of Western Europe and represents $30 \%$ of all HCV [7]. GT-2 is common in West Africa and South America and common also in Japan, some parts of Europe, and North America [3, 7]. GT-4 and GT-6 are common in Central/North Africa and East/ Southern Asia, respectively [3]. GT-4 is the most prevalent in the Middle East and Africa, which is 
responsible for more than $80 \%$ of infections [2]. Previous data on HCV genotypes in Saudi Arabia has shown that the predominant genotypes are GT-4 (52.6\%) and GT-1 (38.6\%), followed by GT-3 $(5.2 \%)$, GT-2 (3.6\%) $[1,2,8]$. HCV genotyping is being used as a gold standard in the study of HCV distribution in society [2]. In addition, HCV genotyping can be used to clarify transmission patterns by applying phylodynamic tools [9]. In Saudi Arabia, there is a high level of GT-4, and only one study was reported from the Qatif region [8, $10,11]$. Hence, this study aimed to examine the distribution of HCV genotypes in Qatif Central Hospital, Saudi Arabia.

\section{PATIENTS AND METHODS}

Study area, design, and period

This is a retrospective study, and data were retrieved from the medical records in Qatif Central Hospital, Qatif, Saudi Arabia, between January 2015 and December 2020.

\section{Study participants}

Only adult patients with positive HCV RNA and documented genotyping were included in the study. Characteristics data included the patient's age, sex, nationality, HCV genotypes.

\section{HCV genotyping analysis}

HCV RNA genotyping was performed by real-time PCR (Abbott diagnostics, Abbott m2000rt, USA).

\section{Statistical analysis}

A statistical package SPSS (SPSS v.23) for Windows was used for the analysis of data and summary statistics. All variables were calculated as frequency and percentage.

\section{Ethical consideration}

The study was conducted according to the guidelines of the Declaration of Helsinki and approved by the Institutional Research Ethic Committee of Qatif Central Hospital (QCH-SREC0244/2021).

\section{RESULTS}

Patients' characteristics

The medical records of 467 adult patients with HCV RNA-positive test results were retrieved from January 2015 to December 2020. Among
Table 1 - Sociodemographic characteristics of HCV patients ( $\mathrm{N}=356)$.

\begin{tabular}{|l|c|c|c|}
\hline Characteristics & Variables & $N$ & $\%$ \\
\hline \multirow{3}{*}{ Nationality } & Saudi & 347 & $(97.5)$ \\
\cline { 2 - 4 } & Non-Saudi & 9 & $(2.5)$ \\
\hline \multirow{3}{*}{ Gender } & Male & 179 & $(50.3)$ \\
\cline { 2 - 4 } & Female & 177 & $(49.7)$ \\
\hline \multirow{4}{*}{ Age } & $<24$ & 17 & $(4.8)$ \\
\cline { 2 - 4 } & $25-49$ & 80 & $(22.5)$ \\
\cline { 2 - 4 } & $50-69$ & 191 & $(53.7)$ \\
\cline { 2 - 4 } & $\geq 70$ & 68 & $(19.1)$ \\
\hline
\end{tabular}

those patients, 111 patients did not have genotypes due to a viral RNA level below the detection limit $(<500 \mathrm{IU} / \mathrm{mL})$, and thus those patients were excluded from the study. Therefore, a total of 356 $\mathrm{HCV}$-infected patients fulfilled the inclusion criteria and were included in further analysis. Of those patients, $179(50.3 \%)$ were males, the majority were Saudi ( $N=347,97.5 \%)$, the median age of the patients was 60 years, of which $191(53.7 \%)$ were between 50 and 69 years old (Table 1 ).

\section{Overall HCV genotypes distribution pattern}

(2015-2020)

Overall, genotype 2 was found in 118 (33.1\%) of the patients, followed by genotype 4 in $92(25.8 \%)$, genotype $1 \mathrm{~B}$ in $62(17.4 \%)$ and genotype $1 \mathrm{~A}$ in 37 $(10.4 \%)$ (Figure 1).

HCV genotypes distribution pattern annually

Table 2 shows the annual percentages of different genotypes. Genotypes 2 and genotype 1B were the most common in 2015, 2018, and 2019 (Figure 2). Genotypes 2 and genotype 4 were the predominant genotypes in 2016, 2017, and 2020 (Figure 2).

Mixed HCV genotypes distribution pattern annually Mixed HCV genotypes 1 and 4 were the most common variants in 2018 ( $\mathrm{N}=3,4.8 \%)$. Only one case of mixed HCV genotype (1 and 6) was detected in 2018 (1.6\%), mixed HCV genotypes (2A and $2 \mathrm{C})$ were seen in two cases in $2016(1.1 \%)$, and one case in 2020 (3.6\%) (Table 2).

HCV genotyping differences between age groups $(<50$ and $\geq 50$ years of age $)$

Table 3 shows the distribution of HCV genotypes in patients 50 years of age and older, and those 
Figure 1 - The proportion of hepatitis $\mathrm{C}$ virus (HCV) genotype in Qatif Central Hospital $(\mathrm{N}=356)$.

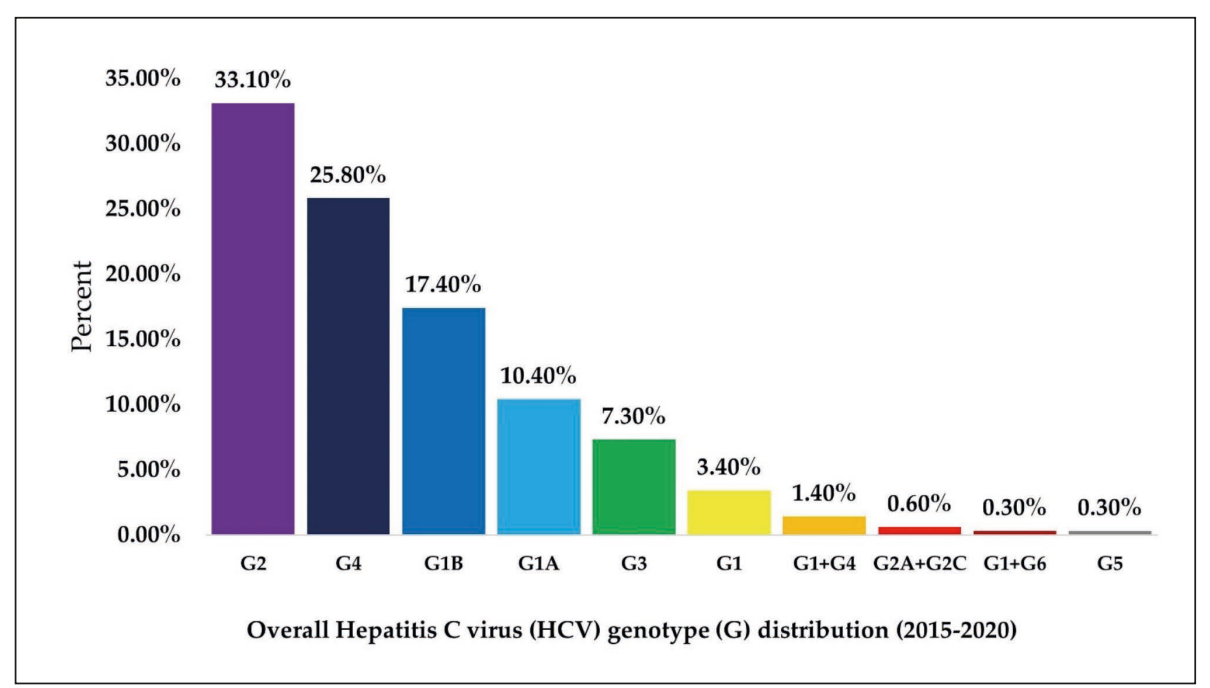

under 50 years of age. Genotype 2 (36.5\% and $25.9 \%$, respectively) and genotype $4(27.5 \%$ and $22.3 \%$, respectively) were the most common genotypes among those patients 50 years of age and older and those under 50 years of age.

\section{DISCUSSION}

It has been recognized that effective control of $\mathrm{HCV}$ infection depends on detection of genotype (GT) distribution, as it predicts the response to therapy and as an integral part of the selection and duration of interferon-free direct-acting antiviral drug (DAA) regimen [3]. Variations in genotype prevalence present challenges to the development of vaccines and therapeutics. The geographic distribution of HCV genotypes varies, and the transmission mode may influence the dominance of specific genotypes $[3,12]$. The study demonstrated the difference in the distribution of hepatitis $\mathrm{C}$ virus genotypes between the age groups of patients 50 years and older and less than 50 years. Genotype $2(36.5 \%$ and $25.9 \%$, respectively) and genotype $4(27.5 \%$ and $22.3 \%$, respectively) are the most frequent genotypes in these patients 50 years and older, and under 50 years of age. These findings are consistent with a Chinese study that found genotype 2 to be more prevalent in HCV patients aged 60 to 69 (12 cases of 31), and the 50-59 age group has the highest HCV infection rate (25.85\%) [13].
Figure 2 - The most common hepatitis $\mathrm{C}$ virus (HCV) genotype distribution annually (2015-2020, $\mathrm{N}=356$ ).

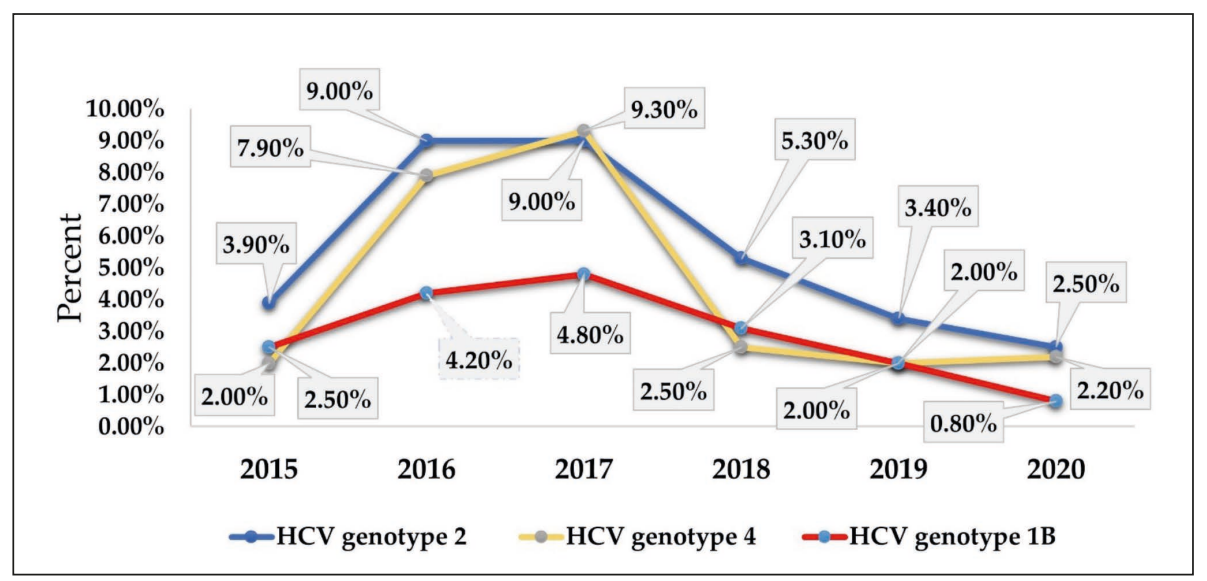


Table 2 - Annual distribution of the hepatitis C virus (HCV) genotype (2015-2020, $\mathrm{N}=356$ ).

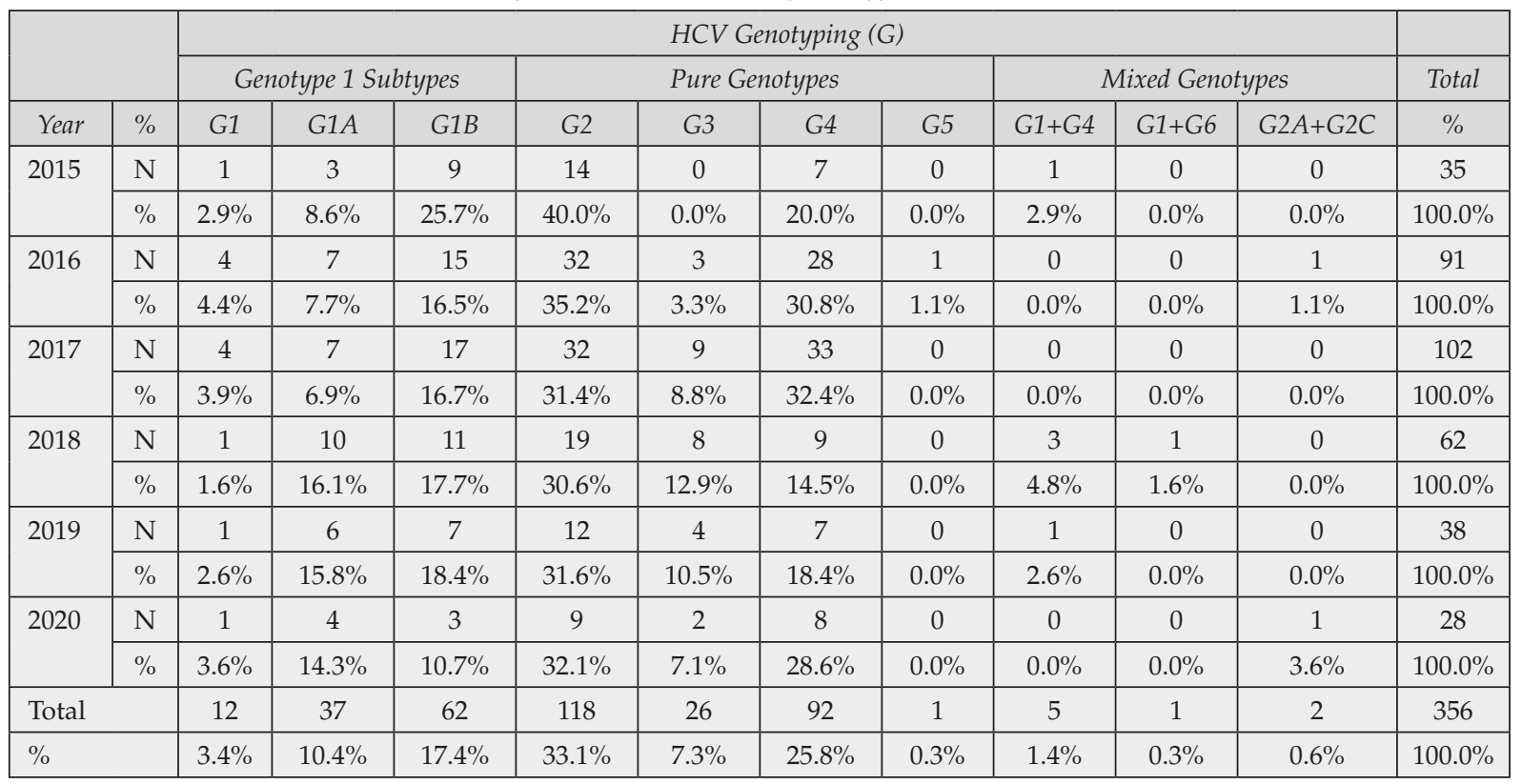

Table 3 - HCV genotyping differences between age groups ( $\mathrm{N}=356)$.

\begin{tabular}{|l|c|c|c|c|}
\hline \multirow{2}{*}{$\begin{array}{c}\text { HCV } \\
\text { Genotyping }\end{array}$} & \multicolumn{2}{|c|}{$<50$} & \multicolumn{2}{c|}{$\geq 50$} \\
\cline { 2 - 5 } G1 & $N$ & $(\%)$ & $N$ & $(\%)$ \\
\hline G1A & 9 & 8.0 & 3 & 1.2 \\
\hline G1B & 23 & 20.5 & 14 & 5.7 \\
\hline G2 & 29 & 10.7 & 50 & 20.5 \\
\hline G3 & 9 & 25.9 & 89 & 36.5 \\
\hline G4 & 25 & 22.3 & 67 & 27.5 \\
\hline G5 & 1 & 0.9 & 0 & 0.0 \\
\hline G1+G4 & 3 & 2.7 & 2 & 0.8 \\
\hline G1+G6 & 0 & 0.0 & 1 & 0.4 \\
\hline G2A+G2C & 1 & 0.9 & 1 & 0.4 \\
\hline Total & 112 & 100.0 & 244 & 100.0 \\
\hline
\end{tabular}

In this study, most chronically HCV-infected individuals were infected with GT-2 and GT-4 (33.1\% and $25.8 \%$ ), respectively. In Saudi Arabia, GT-4 was cited as the most common HCV genotype [2, $9,12]$. In addition, few studies reported a high prevalence of GT-4 across Saudi Arabia [2, 9, 12]. In a previous study from the Qatif region, 20 (39.2\%) of 51 sickle cell patients had HCV GT-2, and GT-3 (25.5\%) had GT-1 [11]. We hypothesize that the high incidence of sickle cell anemia in the Qatif area may be the reason for the high incidence of the GT-2 genotype in these patient populations. These patients may require blood transfusions, making them more likely to develop GT2. Another study in the Eastern Province of Saudi Arabia, Dammam City, showed that 131 people (38\%) have predominant HCV GT-1b [14]. A recent Saudi survey conducted in Jeddah has reported that there is a lower prevalence of GT-2 $(4.1 \%)$ and higher HCV genotype $4(60.7 \%)$ [1]. Because GT4 is very common in Egypt (10\%-15\%) and that many Egyptians work in Saudi Arabia thus it is assumed that GT4 arrived in Saudi Arabia with employees from Africa and the Middle East $[2,7,9]$. This is further supported by the sequencing of the HCV core region [15]. GT-4 has existed in Saudi Arabia for a long time, and the recent migration represents a relatively small source of HCV diversity [9]. According to research data, there is a wide genetic diversity of GT-2 infections in West Africa [16]. During the twentieth century, the prevalence of $\mathrm{HCV}$ containing $\mathrm{HCV}$ subtypes $2 \mathrm{a}, 2 \mathrm{~b}$, and $2 \mathrm{c}$ increased exponentially due to the expansion of parenteral transmission pathways. The newly identified and published $\mathrm{HCV}$ sequence and the origin of these genotypes are of particular importance by increasing the 
chances of an accurate analysis of HCV spread to Saudi Arabia [15]. These time frames led to the discovery that the history of the GT-4d epidemic in Saudi Arabia can be traced back to the 1950s when Saudi Arabian medical services and blood banks were established [2]. At that time, units of blood were primarily imported from France and the United States of America [2].

In the current study, the median age of the patients was 60 years, and $53.7 \%$ were between 50 and 69 years of age. The most recent seroprevalence of HCV among blood donors was $0.04-0.2 \%$. However, HCV was commonly reported among older individuals with a higher age than HBV and HAV [5]. Improved screening and advancing medical care had resulted in decreasing HCV seroprevalence in Saudi Arabia [17, 18].

Several limitations remained. First, this is a onecenter study that evaluated only patients admitted to a Qatif Central Hospital. Consequently, the results may not be representative across the region. Although GT-2 continues to dominate, the distribution and prevalence of hepatitis $C$ virus genotypes are changing in our area mainly due to population increase, increased frequency of patients with hereditary blood diseases, and inbreeding as well.

Further research is needed to understand the epidemiology, risk factors for the differences in the $\mathrm{HCV}$ genotypes and subtypes. Additional data are required for genomic sequences of $\mathrm{HCV}$ isolates from Saudi Arabia's Eastern Provinces to assess the molecular characteristics and epidemiological history. Furthermore, obtaining data from a large-scale population for HCV genotypes can provide important information for healthcare administrators and personnel to monitor and manage HCV infection. HCV infection appears to be equally distributed between genders in this study, which is unusual. Thus, further investigation and research are needed to investigate possible risk factors for HCV infection and determine the HCV genotyping of patients with sickle cell anemia and the gender differences.

In conclusion, this study highlights the hepatitis $\mathrm{C}$ Virus (HCV) genotype distribution pattern in one region of Saudi Arabia. The findings of our study demonstrate a change in the genotypic distribution profile of HCV from genotypes over the six years in patients referred to our hospital. In Qatif city, the most prevalent HCV genotype var- iant distribution pattern is genotype 2, followed by genotype 4 . Perhaps the difference in this genotype distribution can depend on globalization, technological developments, changes in human and social movements.

\section{Authors contributions}

All the authors contributed to the concept, acquisition, and analysis of data, drafting of the article, and critical revision for important intellectual content.

\section{Conflict of interest}

The authors have no conflicts of interest to disclose.

\section{Source of support/funding}

This study was supported by Eitharqatif Committee in Qatif city, Saudi Arabia.

\section{Acknowledgments}

We thank Zahrah Ibrahim A. Al-Koder for collecting the data.

\section{REFERENCES}

[1] Bawazir A, AlGusheri F, Jradi H, et al. Hepatitis C virus genotypes in Saudi Arabia: a future prediction and laboratory profile. Virol J. 2017; 14, 208.

[2] Khan A, Al Balwi M, AlAyyar L, et al. Tracing the epidemic history of hepatitis $C$ virus genotypes in Saudi Arabia. Infect Genet Evol. 2017; 52, 82-8.

[3] Mahmud S, Al-Kanaani Z, Chemaitelly H, et al. Hepatitis $C$ virus genotypes in the Middle East and North Africa: Distribution, diversity, and patterns. J Med Virol. 2018; 90, 131-41.

[4] Fakeeh M, Zaki AM. Hepatitis C: prevalence and common genotypes among ethnic groups in Jeddah, Saudi Arabia. Am J Trop Med Hyg. 1999; 61, 889-92.

[5] Al-Tawfiq JA, Anani A. Profile of viral hepatitis A, B, and $\mathrm{C}$ in a Saudi Arabian hospital. Med Sci Monit Int Med J Exp Clin Res. 2008; 14, CR52-56.

[6] Ngoc CL, Thanh TTT, Lan PTT, et al. Differential prevalence and geographic distribution of hepatitis $C$ virus genotypes in acute and chronic hepatitis $C$ patients in Vietnam. Plos One. 2019; 14, e0212734.

[7] AlMalki WH, Shahid I, Abdalla AN, et al. Virological surveillance, molecular phylogeny, and evolutionary dynamics of hepatitis $C$ virus subtypes $1 \mathrm{a}$ and $4 \mathrm{a}$ isolates in patients from Saudi Arabia. Saudi J Biol Sci. 2021; 28, 1664-77.

[8] Blach S, Zeuzem S, Manns M, et al. Global prevalence and genotype distribution of hepatitis $C$ virus in- 
fection in 2015: a modelling study. Lancet Gastroenterol Hepatol. 2017; 2, 161-76.

[9] Al-Qahtani AA, Baele G, Khalaf N, et al. The epidemic dynamics of hepatitis $C$ virus subtypes 4 a and $4 d$ in Saudi Arabia. Sci Rep. 2017; 7, 44947.

[10] Al-Faleh FZ, Ramia S. Hepatitis C virus (HCV) infection in Saudi Arabia: A review. Ann Saudi Med. 1997; $17,77-82$.

[11] Issa H. Safety of pegylated interferon and ribavirin therapy for chronic hepatitis C in patients with sickle cell anemia. World J Hepatol. 2010; 2, 180-4.

[12] Hazazi A, Almubarak M, Albloui F, et al. Hepatitis C Virus Genotyping in Saudi Population. J US-China Med Sci; 16. Epub ahead of print 28 June 2019. DOI: 10.17265/1548-6648/2019.03.005.

[13] Niu Z, Zhang P, Tong Y. Age and gender distribution of Hepatitis $C$ virus prevalence and genotypes of individuals of physical examination in WuHan, Central China. SpringerPlus. 2016; 5, 1557.

[14] Alzahrani AJ, Dela Cruz DM, Obeid OE, et al. Mo- lecular detection of hepatitis B, hepatitis C, and torque teno viruses in drug users in Saudi Arabia. J Med Virol. 2009; 81, 1343-7.

[15] Shier MK, Iles JC, El-Wetidy MS, et al. Molecular characterization and epidemic history of hepatitis $C$ virus using core sequences of isolates from Central Province, Saudi Arabia. PLoS One. 2017; 12, e0184163.

[16] Candotti D, Temple J, Sarkodie F, et al. Frequent recovery and broad genotype 2 diversity characterize Hepatitis C Virus infection in Ghana, West Africa. J Virol. 2003; 77, 7914-23.

[17] Sallam TA, El-Bingawi HM, Alzahrani KI, et al. Prevalence of hepatitis B and hepatitis C viral infections and impact of control program among blood donors in Al-Baha region, Saudi Arabia. Prevalence. 2020; 9, 56-60.

[18] AlMutairi HH, AlAhmari MM, Al-Zahran BH, et al. Prevalence of serological markers and nucleic acid for blood-borne viral infections in blood donors in Al-Baha, Saudi Arabia. J Infect Dev Ctries. 2016; 10, 619-25. 